\title{
Uniform convergence of multigrid methods for adaptive meshes
}

\author{
Jinbiao $\mathrm{Wu}^{\mathrm{a}}$, Hui Zheng ${ }^{\mathrm{b}, *}$ \\ ${ }^{a}$ School of Mathematical Sciences, Peking University, Beijing 100871, China \\ ${ }^{b}$ School of Mathematics and Statistics, Huazhong University of Science and Technology, Wuhan 430074, \\ China
}

\begin{abstract}
In this paper we study the multigrid methods for adaptively refined finite element meshes. In our multigrid iterations, on each level we only perform relaxation on new nodes and the old nodes whose support of nodal basis function have changed. The convergence analysis of the algorithm is based on the framework of subspace decomposition and subspace correction. In order to decompose the functions from the finest finite element space into each level, a new projection is presented in this paper. Briefly speaking, this new projection can be seemed as the weighted average of the local $L^{2}$ projection. We can perform our subspace decomposition through this new projection by its localization property. Other properties of this new projection are also presented and by these properties we prove the uniform convergence of the algorithm in both $2 \mathrm{D}$ and 3D. We also present some numerical examples to illustrate our conclusion.
\end{abstract}

Keywords: multigrid method, adaptive meshes 2010 MSC: $65 \mathrm{~N} 55,65 \mathrm{~N} 12,65 \mathrm{~F} 10,65 \mathrm{~N} 30$

\section{Introduction}

In this paper we consider multigrid methods for adaptively refined finite element meshes. We consider the following modeling problem: find $u \in H_{0}^{1}(\Omega)$ such that

$$
a(u, v)=(\nabla u, \nabla v)=(f, v), \quad \forall v \in H_{0}^{1}(\Omega)
$$

where $(\cdot, \cdot)$ denotes the $L^{2}(\Omega)$ inner product, $f \in L^{2}(\Omega)$ and $\Omega \in \mathbb{R}^{2}$ is a polygonal domain or $\Omega \in \mathbb{R}^{3}$ is a polyhedral domain.

Adaptive methods are widely used in the finite element methods. Through a posteriori error estimates, adaptive methods provide an efficient way to refine the meshes and increase the efficiency of computation. The convergence of the adaptive method$\mathrm{s}$ are studied in $[1,2,3,4]$ and recently the optimal convergence order for adaptive

\footnotetext{
* Corresponding author Zheng)

Email addresses: jwu@math.pku.edu. cn (Jinbiao Wu), zhenghui@hust.edu. cn (Hui
} 
methods have been proved in [5, 6]. The remaining part to constitute the entire optimal computational complexity is the solving for the discrete linear system arising from the adaptive procedure.

To solve the discrete problems from the adaptive grid, multigrid methods have been widely used. $[7,8]$ developed the the multilevel adaptive technique (MLAT). The fast adaptive composite (FAC) grid method was studied in $[9,10]$. Multigrid methods for adaptive grid were also studied in [11, 12, 13]. Hierarchical basis (HB) methods $[14,15]$ perform relaxations only at the new nodes on each level, it is most economical but the uniform convergence is not guaranteed. To achieve uniform convergence, more relaxations must be added. [16] studied the algorithm that relaxations are performed at the new nodes and all their neighbors (whose basis functions have a support that intersects with the support of basis functions of the new nodes). A more economical way is that relaxations are performed at the new nodes and only their immediate neighbors (whose support of nodal basis function have changed) [17, 18].

[17] studied the newest vertex bisection algorithm for the 2D case, and proved the uniform convergence of the multigrid $V$-cycle algorithm with Gauss-Seidel relaxation performed only on new nodes and their immediate neighbors. In the analysis of [17], Xu-Zikatanov identity [19] is applied and the Scott-Zhang projection [20] is used to decompose the functions in the finite element space. In $2 \mathrm{D}$ cases, when we do a subtraction between two neighboring Scott-Zhang projections, the difference is a function that vanishes on all the nodes except the new nodes and their immediate neighbors. However, in 3D cases, the Scott-Zhang projection no longer has this property. Therefore the decomposition (15) can only be satisfied in 2D case when using Scott-Zhang projections. That is the reason why the conclusion in [17] is hard to be improved to the $3 \mathrm{D}$ cases. The most important contribution in our paper is that we propose a new local projection instead of the Scott-Zhang projection. In 3D cases, this new projection also guarantees the same decomposition 15 as in $2 \mathrm{D}$. Through this new projection, we improve the conclusion in [17] to the 3D cases.

Recently, [18] proved the uniform convergence of the multigrid $V$-cycle algorithm of adaptive meshes in both 2D and 3D cases. They also used the Scott-Zhang projection to decompose the function in the finite element space to apply the Xu-Zikatanov identity. In their algorithm, additional smoothings on each freedom of the finest grid is needed besides the smoothings on new nodes and their immediate neighbors. For the linear elements with this method, the number of smoothings in a problem with degree of freedoms $N$ is about $4 N$. In all these $4 N$ smoothings, $3 N$ smoothings are on new nodes and their immediate neighbors and $N$ smoothings are on each nodes of the finest grid. In this paper, we performing relaxations only on new nodes and their immediate neighbors. Because of the absence of the smoothings on each nodes of the finest grid, the number of smoothings in this algorithm is at most $3 N$. Furthermore, this number is usually less than $3 N$ because the repeated counting of the new nodes' neighbors. By our new projection instead of the Scott-Zhang projection, we also prove the uniform convergence of this algorithm for adaptive meshes in both 2D and 3D cases.

Similar as [17] and [18], our analysis of the convergence is based on the XuZikatanov identity of the framework of subspace correction [21]. In the subspace decomposition, we choose the subspaces to be the node basis function spaces of the new nodes and their immediate neighbors on each level. In order to decompose the 
function in the entire finite element space into each subspace, we first need decompose the function into each level. A special local $L^{2}$ average projection is presented to take place of the Scott-Zhang projection in this paper, and the decomposition on each level is defined to be the difference of this projection between the neighboring two levels. The most important advantage of this projection is that the decomposition in each level only has non-zero values on new nodes and their immediate neighbors, both in 2D and 3D cases (Lemma 3.1). Then on each level we can continue decomposing the function into the node basis functions of new nodes and their immediate neighbors. Some other properties of this projection is proposed, such as the stability of the $L^{2}$ norm (Lemma 3.2, Lemma 3.3), the stability of the energy norm (Lemma 3.4, Lemma 3.5), the approximation property (Lemma 3.6) and the stability of the multilevel decomposition (Lemma 3.7). The stability of the multilevel decomposition is important for our theoretical analysis. The proof of Lemma 3.7 relies on a relation between the sequence of the adaptive meshes and a sequence of nested quasi-uniform meshes, and this method is inspired from [17]. By these properties and the similar analysis developed in [22, 23], we can prove the the uniform convergence of our algorithm. Furthermore, we consider the discretization of high order Lagrange element and also prove the uniform convergence through the new projection.

In this paper, we denote $x_{1} \lesssim y_{1}$ and $x_{2} \gtrsim y_{2}$ if there exist generic positive constants $C_{1}$ and $C_{2}$ independent of any other parameters related to the mesh, the spaces and, in particular, the coefficients such that $x_{1} \leqslant C_{1} y_{1}$ and $x_{2} \geqslant C_{2} y_{2}$. Furthermore, we denote $x_{3} \simeq y_{3}$ if $x_{3} \lesssim y_{3}$ and $x_{3} \gtrsim y_{3}$. The general notations of the Sobolev space are also used. For some domain $\omega$, the $L^{2}(\omega)$ norm and the $H^{1}(\omega)$ semi-norm are denoted by $\|\cdot\|_{0, \omega}$ and $|\cdot|_{1, \omega}$ respectively. Specially, when $\omega$ is the entire domain $\Omega$, we may omit the tag of domain and use the notation $\|\cdot\|_{0}$ and $|\cdot|_{1}$. Additionally, we use the notation $\|\cdot\|_{a}=|\cdot|_{1}$.

The rest of this paper is organized as follows. In Section 2, we describe our adaptive meshes with some settings, and give the subspace correction algorithm. In Section 3 , we introduce our new projection and prove some properties of this projection. In Section 4 , we prove the uniform convergence of the multigrid methods for the adaptive meshes. In Section 5, we consider the discretization of high order Lagrange element and prove its uniform convergence. In Section 6, we present some numerical results to illustrate our theoretic results.

\section{Adaptive meshes and the subspace correction algorithm}

\subsection{Adaptive meshes}

Suppose we have a sequence of nested triangulations $\mathcal{T}_{k}=\left\{\tau_{k}^{i}\right\}(1 \leq k \leq L, 1 \leq$ $\left.i \leq p_{k}\right)$ of $\Omega$, where $\tau_{k}^{i}$ is simplex. Now we denote by $V_{k}\left(\subset H_{0}^{1}(\Omega)\right)$ the finite element space of the continuous piecewise linear functions associated with triangulation $\mathcal{T}_{k}$. Then

$$
V_{1} \subset \cdots \subset V_{k} \subset \cdots \subset V_{L},
$$

and we set $V_{L}=V$. Then the discrete problem is: find $u \in V$ such that

$$
a(u, v)=(\nabla u, \nabla v)=(f, v), \quad \forall v \in V .
$$


According to the adaptive finite element method, after each refinement we obtain a new finest triangulation $\mathcal{T}_{L}$. Then we need solve (2) after each refinement. However, the focus of this paper is to solve the discrete problem (2) by multigrid method, and we only need discuss the solving of (2) for a certain level $L$.

Notice that we do not restrict the generation of the sequence $\mathcal{T}_{k}$. Besides the finest triangulation $\mathcal{T}_{L}$, which is generated from the actual process of adaptively refinement, other $\mathcal{T}_{k}$ can be chosen arbitrarily. The only requirement on $\mathcal{T}_{k}$ is that they are nested and satisfy the following three assumptions (A1), (A2) and (A3). Of course, the nature choice of $\mathcal{T}_{k}$ is the triangulation after $k$-th refinement. Another method is to generate $\mathcal{T}_{k}$ directly from the finest triangulations $\mathcal{T}_{L}$ through some course of coarsing $[18,24]$.

First we propose the two basic assumptions (A1) and (A2):

(A1) The triangulation $\mathcal{T}_{k}$ is conforming, $\forall 1 \leq k \leq L$.

(A2) The triangulation $\mathcal{T}_{k}$ is shape regular, i.e., $h\left(\tau_{k}^{i}\right) / \rho\left(\tau_{k}^{i}\right)<C, \forall 1 \leq k \leq L, 1 \leq$ $i \leq p_{k}$, where $C$ is independent of $k$ and $i, h\left(\tau_{k}^{i}\right)$ and $\rho\left(\tau_{k}^{i}\right)$ denote the diameter of $\tau_{k}^{i}$ and the diameter of the inscribed circle or sphere of $\tau_{k}^{i}$, respectively.

These two assumptions are very basic in the settings in finite element method. In adaptive finite element method, many usual adaptive refinements satisfy these two assumptions. For example, in the 2D case the newest vertex bisection method satisfy (A1) and (A2); in the 3D case we give an example of the bisection method which satisfies (A1) and (A2) [25, 26]. This example can be seen as a variant of the newest bisection method in the $2 \mathrm{D}$ case. Each tetrahedron element is ordered of the vertices and has a type index $s$ with $s \in\{0,1,2,3\}$. Then when a ordered tetrahedron $\tau=\left(x_{0}, x_{1}, x_{2}, x_{3}\right)_{s}$ is refined, the children are the ordered tetrahedrons

$$
\left(x_{0}, \frac{x_{0}+x_{3}}{2}, x_{1}, \cdots, x_{s}, x_{s+1}, \cdots, x_{2}\right)_{(s+1) \bmod 3}
$$

and

$$
\left(x_{0}, \frac{x_{0}+x_{3}}{2}, x_{1}, \cdots, x_{s}, x_{2}, \cdots, x_{s+1}\right)_{(s+1) \bmod 3} .
$$

This rule of refinement and some proper initial triangulation can assure the assumptions (A1) and (A2).

Before the introduction of (A3), we give some notation on our meshes. We denote by $M_{k}=\left\{x_{k}^{i}, i=1, \cdots, m_{k}\right\}$ the set of interior nodes of $\mathcal{T}_{k}$, and define

$$
\Omega_{k}^{i}=\bigcup_{\tau \in \mathcal{T}_{k}, x_{k}^{i} \in \tau} \tau,
$$

which is the support of nodal basis function of node $x_{k}^{i}$ (by convention, all the elements are deemed to be closed sets). For $z=x_{k}^{i}$, we also denote $\Omega_{k}^{z}=\Omega_{k}^{i}$. We denote by $h_{k, i}$ the diameter of $\Omega_{k}^{i}$. Then we give

(A3) There exists a nested quasi-uniform triangulations $\widehat{\mathcal{T}}_{l}=\left\{\widehat{\tau}_{l}^{j}\right\}(1 \leq l, 1 \leq j \leq$ $\left.\widehat{n}_{l}\right)$ of $\Omega$ with the diameter $h_{l}$, which satisfies the following property: for any $z=x_{k}^{i}\left(1 \leq k \leq L, 1 \leq i \leq m_{k}\right)$, there exists an integer $l$ satisfies 
(i) $h_{l} \leq C h_{k, i}$, where $C$ is independent of $k$ and $i$.

(ii) For all $\tau \in \mathcal{T}_{k-1}, \tau \cap \Omega_{k-1}^{z} \neq \emptyset$, there exists $1 \leq j \leq \widehat{n}_{l}$ such that $\tau \subset \widehat{\tau}_{l}^{j}$.

Roughly speaking, (A3) means the quasi-uniform meshes $\widehat{\mathcal{T}}_{l}$ can "exactly" "cover" the meshes $\mathcal{T}_{k}$ on every localized region. It guarantees an important formulation (10), which is fundamental in our theoretical analysis. In many cases (A3) holds. For example, when $\mathcal{T}_{k}$ is according to the process of adaptively refinement, the newest bisection method in the 2D case satisfies (A3); in a general bisection method, when the condition "each triangulation in the sequence of the uniform refinement from the initial triangulation is conforming" is satisfied, we can choose the nested quasi-uniform triangulations $\widehat{\mathcal{T}}_{l}$ to be this sequence of the uniform refinement. In fact, the example of bisection we give before (A3) satisfies the above condition while the coarsest partition is conforming and a so-called matching condition is satisfied [26]. Hence, the example of the bisection method in 3D we present above can also assure the assumptions (A3).

\subsection{Multigrid algorithm and subspace correction framework}

We define $N_{k}$ to be the new nodes and their immediate neighboring nodes on the $k$-th mesh, where the immediate neighboring nodes mean that the support of the node's nodal basis changes from the $k-1$-th mesh to the $k$-th mesh. Then $N_{k}$ is a subset of $M_{k}$, we define

$$
N_{k}=\left\{y_{k}^{i}, i=1, \cdots, n_{k}\right\},
$$

and we define $V_{k}^{i}=\operatorname{span}\left\{\phi_{k}^{i}\right\}$ and each $\phi_{k}^{i}$ is the usual finite element basis function which is 1 at the node $y_{k}^{i}$ and 0 at all the other nodes. Notice that we have

$$
V_{k}=V_{k-1}+\sum_{i=1}^{n_{k}} V_{k}^{i},
$$

therefore we have

$$
V=\sum_{k=1}^{L} \sum_{i=1}^{n_{k}} V_{k}^{i} .
$$

Our multigrid algorithm performs relaxations at each node of $N_{k}$ on the $k$-th mesh. According to Algorithm 2.1, the Gauss-seidel relaxation is chosen.

Now we introduce the abstract framework of the subspace decomposition and the subspace correction to analyze our multigrid algorithm [21]. We assume that $V$ is decomposed into a sum of subspaces $\left\{W_{i}\right\}_{i=1}^{J}$, i.e.,

$$
V=\sum_{i=1}^{J} W_{i} .
$$

A successive subspace correction algorithm with exact subspace solvers is presented as follows. 
Algorithm 2.1 (SSC). Let the initial guess $u^{0} \in V$ be given,

for $l=1, \cdots$

$$
\begin{aligned}
& u_{0}^{l-1}=u^{l-1} \\
& \text { for } i=1, \cdots, J \\
& \quad u_{i}^{l-1}=u_{i-1}^{l-1}+e_{i} \text { where } e_{i} \in W_{i} \text { satisfies } \\
& \qquad a\left(e_{i}, w_{i}\right)=\left(f, w_{i}\right)-a\left(u_{i-1}^{l-1}, w_{i}\right), \quad \forall w_{i} \in W_{i}
\end{aligned}
$$

endfor

$u^{l}=u_{J}^{l-1}$

\section{endfor}

Notice that when the decomposition (5) is chosen as (4) with the order $V_{1}^{1}, V_{1}^{2}$, $\cdots, V_{1}^{n_{1}}, V_{2}^{1}, V_{2}^{2}, \cdots, V_{2}^{n_{2}}, \cdots \cdots, V_{L}^{1}, V_{L}^{2}, \cdots, V_{L}^{n_{L}}$, Algorithm 2.1 is exactly the multigrid algorithm performs one step Gauss-seidel relaxation at each node of $N_{k}$ on the $k$-th mesh. With Algorithm 2.1, we obtain

$$
\left(u-u^{l}\right)=\left(I-P_{W_{J}}\right) \cdots\left(I-P_{W_{1}}\right)\left(u-u^{l-1}\right),
$$

where $P_{W_{i}}$ denotes the projection onto $W_{i}$ with respect to the $a$-inner product. Therefore we can define the error transfer operator

$$
E=\left(I-P_{W_{J}}\right) \cdots\left(I-P_{W_{1}}\right),
$$

and with this notation we have

$$
\left(u-u^{l}\right)=E^{l}\left(u-u^{0}\right) .
$$

In the follows, we will use $\|E\|_{a}$ to evaluate the convergence rate of Algorithm 2.1, where

$$
\|E\|_{a}^{2}=\sup _{v \in V} \frac{\|E v\|_{a}^{2}}{\|v\|_{a}^{2}}=\sup _{v \in V} \frac{a(E v, E v)}{a(v, v)},
$$

and by Xu-Zikatanov identity [19], we know that $\|E\|_{a}^{2}=1-\frac{1}{K}$, where

$$
K=\sup _{v \in V} \inf _{\sum w_{j}=v} \frac{\sum_{j=1}^{J}\left\|P_{j} \sum_{i=j}^{J} w_{i}\right\|_{a}^{2}}{\|v\|_{a}^{2}} .
$$

In our actual problem, we apply the decomposition (4), then

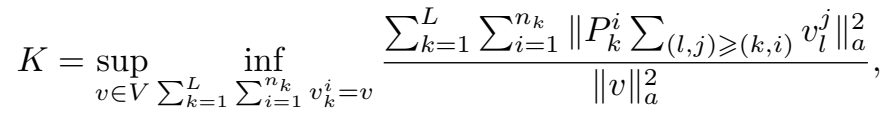

where $(l, j) \geqslant(k, i)$ means $l>k$ or $l=k, j \geqslant i$. By the projection presented in Section 3, we give a decomposition $v=\sum_{k=1}^{L} \sum_{i=1}^{n_{k}} v_{k}^{i}$ for all $v \in V$ and by this decomposition we can prove $K \lesssim 1$, then we obtain the uniform convergence of our algorithm (Section 4). 


\section{A weighted average local $L^{2}$ projection}

In this section we introduce a new projection $\Pi_{k}$ from $L^{2}(\Omega)$ to $V_{k}$. Although we only deal with the functions in $H_{0}^{1}(\Omega)$, this new projection can be defined more generally on $L^{2}(\Omega)$. Note that $V_{k}$ is the linear finite element space, therefore we need only define the value of $\Pi_{k} v$ on each node of our triangulation. An important property we expect of the projection $\Pi_{k}$ is "localization", i.e., the value of $\Pi_{k} v$ on a node $z$ only relies on the value of $v$ on a neighboring domain of $z$. The pointwise interpolation is not valid because the function in $H_{0}^{1}(\Omega)$ has no pointwise value in two or more dimensions. A widely used localized projection is the Scott-Zhang interpolation [20]. Another method to obtain a localized projection is to use the local $L^{2}$ projection on each element. However, the local $L^{2}$ projection on two neighboring elements may not be equal on their common node. For continuity, a natural idea is that we calculate the average value on each node. Unfortunately, the projection according to the usual average value on each node does not satisfy Lemma 3.1. In this paper we propose a new projection according to a weighted average value on each node. The weight is simply set to be the measure of the relevant element, where the measure of an element is the area of the element in $2 \mathrm{D}$ and is the volume of the element in 3D. The precise definition of $\Pi_{k}$ is as follows.

For all nodes $z \in M_{k}$, we define

$$
\Pi_{k} v(z)=\frac{\sum_{\tau \in \mathcal{T}_{k}, \tau \ni z} \operatorname{meas}(\tau) \cdot Q_{\tau} v(z)}{\sum_{\tau \in \mathcal{T}_{k}, \tau \ni z} \operatorname{meas}(\tau)}, \quad \forall v \in L^{2}(\Omega),
$$

where meas $(\tau)$ denotes the measure of $\tau$, and the local $L^{2}$ projection $Q_{\tau}$ from $L^{2}(\Omega)$ to $\left.V_{k}\right|_{\tau}$ is defined as follows:

$$
\left(Q_{\tau} v, w\right)_{L^{2}(\tau)}=(v, w)_{L^{2}(\tau)}, \quad \forall v \in L^{2}(\Omega),\left.w \in V_{k}\right|_{\tau} .
$$

Obviously, $\Pi_{k}$ and $\Pi_{k-1}$ have no difference at the location where the mesh is not refined from the $k$ - 1-th level to the $k$-th level. Furthermore, we find that $\Pi_{k}$ and $\Pi_{k-1}$ are equal at the nodes whose support of nodal basis function have not changed. Then we have the following lemma:

Lemma 3.1. $\Pi_{k} v-\Pi_{k-1} v \in \sum_{i=1}^{n_{k}} V_{k}^{i}, \forall v \in L^{2}(\Omega)$.

PROOF. We only need prove that $\forall z \in M_{k} \backslash N_{k}, \Pi_{k} v(z)=\Pi_{k-1} v(z)$. Suppose $z$ is contained in some $\tau_{k-1} \in \mathcal{T}_{k-1}$. If all these $\tau_{k-1}$ are not refined from the $k-1$-th level to the $k$-th level, then $\Pi_{k} v(z)=\Pi_{k-1} v(z)$ is obvious. Now we suppose some $\tau_{k-1}$ has been refined into two elements $\tau_{k}^{1}$ and $\tau_{k}^{2}$ in the $k$-th level.

We introduce the dual basis function $\varphi_{z}\left(\left.\in V_{k-1}\right|_{\tau_{k-1}}\right)$ of the node $z$ on $\tau_{k-1}$ such that

$$
\left(w, \varphi_{z}\right)_{L^{2}\left(\tau_{k-1}\right)}=\operatorname{meas}\left(\tau_{k-1}\right) \cdot w(z), \quad \forall w \in V_{k-1},
$$

and similarly we introduce the dual basis function $\varphi_{z}^{1}\left(\left.\in V_{k}\right|_{\tau_{k}^{1}}\right), \varphi_{z}^{2}\left(\left.\in V_{k}\right|_{\tau_{k}^{2}}\right)$ of the node $z$ on $\tau_{k}^{1}, \tau_{k}^{2}$ such that

$$
\left(w, \varphi_{z}^{1}\right)_{L^{2}\left(\tau_{k}^{1}\right)}=\operatorname{meas}\left(\tau_{k}^{1}\right) \cdot w(z), \quad \forall w \in V_{k},
$$




$$
\left(w, \varphi_{z}^{2}\right)_{L^{2}\left(\tau_{k}^{2}\right)}=\operatorname{meas}\left(\tau_{k}^{2}\right) \cdot w(z), \quad \forall w \in V_{k} .
$$

An advantage of the above dual basis functions is the following relationships:

$$
\varphi_{z}^{1}=\left.\varphi_{z}\right|_{\tau_{k}^{1}}, \varphi_{z}^{2}=\left.\varphi_{z}\right|_{\tau_{k}^{2}} .
$$

In fact, in the $2 D$ case all these above dual basis functions are 9 at $z$ and -3 at other nodes; in $3 D$ case they are 16 at $z$ and -4 at other nodes; then we obtain the relationships (9).

Using these dual basis functions, we have

$$
\begin{aligned}
\operatorname{meas}\left(\tau_{k-1}\right) \cdot Q_{\tau_{k-1}} v(z) & =\left(Q_{\tau_{k-1}} v, \varphi_{z}\right)_{L^{2}\left(\tau_{k-1}\right)} \\
& =\left(v, \varphi_{z}\right)_{L^{2}\left(\tau_{k-1}\right)} \\
& =\left(v, \varphi_{z}^{1}\right)_{L^{2}\left(\tau_{k}^{1}\right)}+\left(v, \varphi_{z}^{2}\right)_{L^{2}\left(\tau_{k}^{2}\right)} \\
& =\left(Q_{\tau_{k}^{1}} v, \varphi_{z}^{1}\right)_{L^{2}\left(\tau_{k}^{1}\right)}+\left(Q_{\tau_{k}^{2}} v, \varphi_{z}^{2}\right)_{L^{2}\left(\tau_{k}^{2}\right)} \\
& =\operatorname{meas}\left(\tau_{k}^{1}\right) \cdot Q_{\tau_{k}^{1}} v(z)+\operatorname{meas}\left(\tau_{k}^{2}\right) \cdot Q_{\tau_{k}^{2}} v(z)
\end{aligned}
$$

Notice that the above equality holds for any $\tau_{k-1}$ that has been refined, then the numerator of (8) for the $k-1$-th level and the $k$-th level are equal. Therefore we obtain $\Pi_{k} v(z)=\Pi_{k-1} v(z)$.

Notice that the above lemma guarantees our decomposition (15) in both 2D and 3D cases, while the Scott-Zhang interpolation projection may not guarantee the decomposition (15) in the 3D case. That is the crucial reason we propose this new interpolation projection.

Next we present some basic properties of the new projection $\Pi_{k}$, including the $L^{2}$ norm stability, the energy norm stability, the approximation property. These properties commonly exist for some local interpolation projections, such as the local $L^{2}$ projection, the average local $L^{2}$ projection, the Scott-Zhang projection and so on. Naturally, we intend to check these basic properties for $\Pi_{k}$.

First we prove the $L^{2}$ norm stability of $\Pi_{k}$. For any element $\tau_{k} \in \mathcal{T}_{k}$, we define

$$
\Omega\left(\tau_{k}\right)=\bigcup_{\tau \in \mathcal{T}_{k}, \tau_{k} \cap \tau \neq \emptyset} \tau .
$$

Then only the value of $v$ on $\Omega\left(\tau_{k}\right)$ can influence the value of $\Pi_{k} v$ on $\tau_{k}$. Naturally, we have the local $L^{2}$ norm stability:

Lemma 3.2. $\left\|\Pi_{k} v\right\|_{0, \tau_{k}} \lesssim\|v\|_{0, \Omega\left(\tau_{k}\right)}, \forall v \in L^{2}(\Omega)$.

Proof. Denote by $\mathcal{N}(\tau)$ the set of all nodes of $\tau$. Then

$$
\begin{aligned}
\left\|\Pi_{k} v\right\|_{0, \tau_{k}}^{2} & \lesssim \operatorname{meas}\left(\tau_{k}\right) \cdot \sum_{z \in \mathcal{N}\left(\tau_{k}\right)} \Pi_{k} v^{2}(z) \\
& \lesssim \sum_{z \in \mathcal{N}\left(\tau_{k}\right)}\|v\|_{0, \Omega_{k}^{z}}^{2} \\
& \lesssim\|v\|_{0, \Omega\left(\tau_{k}\right)}^{2} .
\end{aligned}
$$


From the local $L^{2}$ norm stability, obviously we obtain the $L^{2}$ norm stability on $\Omega$ by finite overlapping.

Lemma 3.3. $\left\|\Pi_{k} v\right\|_{0, \Omega} \lesssim\|v\|_{0, \Omega}, \forall v \in L^{2}(\Omega)$.

PROOF.

$$
\begin{aligned}
\left\|\Pi_{k} v\right\|_{0, \Omega}^{2} & =\sum_{\tau_{k} \in \mathcal{T}_{k}}\left\|\Pi_{k} v\right\|_{0, \tau_{k}}^{2} \\
& \lesssim \sum_{\tau_{k} \in \mathcal{T}_{k}}\|v\|_{0, \Omega\left(\tau_{k}\right)}^{2} \\
& \lesssim\|v\|_{0, \Omega}^{2} .
\end{aligned}
$$

Next, we prove the local energy norm stability between $\Pi_{k} v$ on $\tau_{k}$ and $v$ on $\Omega\left(\tau_{k}\right)$ by some usual scaling arguments.

Lemma 3.4. $\left|\Pi_{k} v\right|_{1, \tau_{k}} \lesssim|v|_{1, \Omega\left(\tau_{k}\right)}, \forall v \in H_{0}^{1}(\Omega)$.

ProOF. We discuss the $2 D$ case and the proof in $3 D$ is similar. Suppose the piecewise affine transformation $F$ maps $\tau_{k}$ to the reference element $\widehat{\tau_{k}}$ and maps $\Omega\left(\tau_{k}\right)$ to the reference elements $\widehat{\Omega\left(\tau_{k}\right)}$. We maps all the $\Omega\left(\tau_{k}\right)$ which contains the same number of elements to a same reference elements $\widehat{\Omega\left(\tau_{k}\right)}$. Then the kind of the reference elements $\widehat{\Omega\left(\tau_{k}\right)}$ is finite. Then for any constant function $c$, we have ( $d$ is the dimension of the space and here $d=2$ )

$$
\begin{aligned}
\left|\Pi_{k} v\right|_{1, \tau_{k}}^{2} & \lesssim h_{\tau_{k}}^{d-2}\left|\widehat{\Pi_{k} v}\right|_{1, \widehat{\tau_{k}}}^{2} \\
& =h_{\tau_{k}}^{d-2}\left|\Pi_{k} \widehat{(v+c)}\right|_{1, \widehat{\tau_{k}}}^{2} \\
& \left.\lesssim h_{\tau_{k}}^{d-2}\left(\widehat{\Pi_{k}} \widehat{(v+c)^{2}}(\widehat{A})+\Pi_{k} \widehat{(v+c)}\right)^{2}(\widehat{B})+\Pi_{k} \widehat{(v+c)^{2}}(\widehat{C})\right) \\
& =h_{\tau_{k}}^{d-2}\left(\Pi_{k}(v+c)^{2}(A)+\Pi_{k}(v+c)^{2}(B)+\Pi_{k}(v+c)^{2}(C)\right) \\
& \lesssim h_{\tau_{k}}^{-2}\|v+c\|_{0, \Omega\left(\tau_{k}\right)}^{2} \\
& \lesssim h_{\tau_{k}}^{d-2}\|\widehat{v}+\widehat{c}\|_{0, \widehat{\Omega\left(\tau_{k}\right)}}^{2} \\
& \lesssim h_{\tau_{k}}^{d-2}\|\widehat{v}+\widehat{c}\|_{1, \widehat{\Omega\left(\tau_{k}\right)}}^{2}
\end{aligned}
$$

where $A, B, C$ is the three vertices of $\tau_{k}$. Because

$$
\begin{aligned}
\inf _{c}\|\widehat{v}+\widehat{c}\|_{1, \widehat{\Omega\left(\tau_{k}\right)}}^{2} & \lesssim|\widehat{v}|_{1, \widehat{\Omega\left(\tau_{k}\right)}}^{2} \\
& \lesssim h_{\tau_{k}}^{2-d}|v|_{1, \Omega\left(\tau_{k}\right)}^{2},
\end{aligned}
$$

then we have

$$
\left|\Pi_{k} v\right|_{1, \tau_{k}} \lesssim|v|_{1, \Omega\left(\tau_{k}\right)} .
$$

From the above lemma, we also have the global stability for the energy norm.

Lemma 3.5. $\left|\Pi_{k} v\right|_{1, \Omega} \lesssim|v|_{1, \Omega}, \forall v \in H_{0}^{1}(\Omega)$. 
PROOF.

$$
\begin{aligned}
\left|\Pi_{k} v\right|_{1, \Omega}^{2} & =\sum_{\tau_{k} \in \mathcal{T}_{k}}\left|\Pi_{k} v\right|_{1, \tau_{k}}^{2} \\
& \lesssim \sum_{\tau_{k} \in \mathcal{T}_{k}}|v|_{1, \Omega\left(\tau_{k}\right)}^{2} \\
& \lesssim|v|_{1, \Omega}^{2} .
\end{aligned}
$$

Now we give the approximation property of the projection $\Pi_{k}$. It also can be obtained by usual scaling arguments.

Lemma 3.6. $\left\|v-\Pi_{k} v\right\|_{0, \tau_{k}} \lesssim h_{\tau_{k}}|v|_{1, \Omega\left(\tau_{k}\right)}, \forall v \in H_{0}^{1}(\Omega)$.

ProOF. We discuss the $2 D$ case and the proof in $3 D$ is similar. Suppose the piecewise affine transformation $F$ maps $\tau_{k}$ to the reference element $\widehat{\tau_{k}}$ and maps $\Omega\left(\tau_{k}\right)$ to the reference elements $\widehat{\Omega\left(\tau_{k}\right)}$. We maps all the $\Omega\left(\tau_{k}\right)$ which contains the same number of elements to a same reference elements $\widehat{\Omega\left(\tau_{k}\right)}$. Then the kind of the reference elements $\widehat{\Omega\left(\tau_{k}\right)}$ is finite. Then for any constant function $c$, we have ( $d$ is the dimension of the space and here $d=2$ )

$$
\begin{aligned}
& \left\|v-\Pi_{k} v\right\|_{0, \tau_{k}}^{2} \lesssim h_{\tau_{k}}^{d}\left\|\widehat{v}-\widehat{\Pi_{k} v}\right\|_{0, \widehat{\tau_{k}}}^{2} \\
& =h_{\tau_{k}}^{d}\left\|\widehat{v}+\widehat{c}-\Pi_{k} \widehat{(v+c)}\right\|_{0, \widehat{\tau_{k}}}^{2} \\
& \left.\lesssim h_{\tau_{k}}^{d}\left(\|\widehat{v}+\widehat{c}\|_{0, \widehat{\tau_{k}}}^{2}\right)+\left\|\widehat{\Pi_{k}(\widehat{v+c})}\right\|_{0, \widehat{\tau_{k}}}^{2}\right) \\
& \left.\lesssim h_{\tau_{k}}^{d}\left(\|\widehat{v}+\widehat{c}\|_{0, \widehat{\tau_{k}}}^{2}+\Pi_{k} \widehat{(v+c)^{2}}(\widehat{A})+\Pi_{k} \widehat{(v+c)^{2}}(\widehat{B})+\Pi_{k} \widehat{(v+c)}\right)^{2}(\widehat{C})\right) \\
& =h_{\tau_{k}}^{d}\left(\|\widehat{v}+\widehat{c}\|_{0, \widehat{\tau_{k}}}^{2}+\Pi_{k}(v+c)^{2}(A)+\Pi_{k}(v+c)^{2}(B)+\Pi_{k}(v+c)^{2}(C)\right) \\
& \lesssim h_{\tau_{k}}^{d}\left(\|\widehat{v}+\widehat{c}\|_{0, \widehat{\tau_{k}}}^{2}+h_{\tau_{k}}^{-d}\|v+c\|_{0, \Omega\left(\tau_{k}\right)}^{2}\right) \\
& \lesssim h_{\tau_{k}}^{d}\left(\|\widehat{v}+\widehat{c}\|_{0, \widehat{\tau_{k}}}^{2}+\|\widehat{v}+\widehat{c}\|_{0, \widehat{\Omega\left(\tau_{k}\right)}}^{2}\right) \\
& \lesssim h_{\tau_{k}}^{d}\left(\|\widehat{v}+\widehat{c}\|_{1, \widehat{\Omega\left(\tau_{k}\right)}}^{2}\right),
\end{aligned}
$$

where $A, B, C$ is the three vertices of $\tau_{k}$. Because

$$
\begin{aligned}
\inf _{c}\|\widehat{v}+\widehat{c}\|_{1, \widehat{\Omega\left(\tau_{k}\right)}}^{2} & \lesssim|\widehat{v}|_{1, \widehat{\Omega\left(\tau_{k}\right)}}^{2} \\
& \lesssim h_{\tau_{k}}^{2-d}|v|_{1, \Omega\left(\tau_{k}\right)}^{2},
\end{aligned}
$$

then we have

$$
\left\|v-\Pi_{k} v\right\|_{0, \tau_{k}} \lesssim h_{\tau_{k}}|v|_{1, \Omega\left(\tau_{k}\right)} .
$$

Finally, we discuss the stability of the multilevel decomposition for projection $\Pi_{k}$, which is important for our theoretical analysis. We try to transform the problem to the nested quasi-uniform meshes which is described in assumption (A3), then apply the stability of the multilevel decomposition for the general $L^{2}$ projection. We suppose the finite element spaces according to the nested quasi-uniform meshes in (A3) are

$$
\widehat{V}_{1} \subset \widehat{V}_{2} \subset \cdots,
$$


and we denote by $h_{l}$ the element scale of $\widehat{V}_{l}$ and denote by $\widehat{Q}_{l}$ the general $L^{2}$ projection from $L^{2}(\Omega)$ to $\widehat{V}_{l}$.

For any $\Omega_{k}^{i}$, we define by $s(k, i)$ the integer $l$ in (A3). From (A3), the meshes of $\widehat{V}_{s(k, i)}$ is coarser than $V_{k}$ and $V_{k-1}$ nearby $x_{k}^{i}$, hence we have

$$
\left.\widehat{Q}_{s(k, i)} v\right|_{\Omega_{k}^{i}}=\left.\Pi_{k} \widehat{Q}_{s(k, i)} v\right|_{\Omega_{k}^{i}}=\left.\Pi_{k-1} \widehat{Q}_{s(k, i)} v\right|_{\Omega_{k}^{i}} .
$$

(10) gives a bridge to connect the adaptive meshes and the quasi-uniform meshes, allow us to transform the problem on the adaptive meshes to the quasi-uniform meshes. The lemma on the stability of the multilevel decomposition is as follows.

Lemma 3.7. For any $v \in H_{0}^{1}(\Omega)$, we have

$$
\begin{gathered}
\sum_{k=1}^{L} \sum_{i=1}^{n_{k}} h_{k, i}^{-2}\left\|\left(\Pi_{k}-\Pi_{k-1}\right) v\right\|_{0, \Omega_{k}^{i}}^{2} \lesssim\|v\|_{a}^{2}, \\
\sum_{k=1}^{L} \sum_{i=1}^{n_{k}} h_{k, i}^{-2}\left\|v-\Pi_{k} v\right\|_{0, \Omega_{k}^{i}}^{2} \lesssim\|v\|_{a}^{2}, \\
\sum_{k=1}^{L}\left|\Pi_{k} v-\Pi_{k-1} v\right|_{1}^{2} \lesssim|v|_{1}^{2} .
\end{gathered}
$$

PROOF. First we prove the following inequality:

$$
\sum_{k=1}^{L} \sum_{i=1}^{n_{k}} h_{k, i}^{-2}\left\|v-\widehat{Q}_{s(k, i)} v\right\|_{0, \widetilde{\Omega}_{k}^{i}}^{2} \lesssim\|v\|_{a}^{2},
$$

where

$$
\widetilde{\Omega}_{k}^{i}=\bigcup_{\tau \in \mathcal{T}_{k}, \tau \cap \Omega_{k}^{i} \neq \emptyset} \tau .
$$

Because of the finite overlapping, we have $\sum_{s(k, i)=l}\left\|v-\widehat{Q}_{l} v\right\|_{0, \widetilde{\Omega}_{k}^{i}}^{2} \lesssim\left\|v-\widehat{Q}_{l} v\right\|_{0, \Omega}^{2}$, then

$$
\begin{aligned}
& \sum_{k=1}^{L} \sum_{i=1}^{n_{k}} h_{k, i}^{-2}\left\|v-\widehat{Q}_{s(k, i)} v\right\|_{0, \widetilde{\Omega}_{k}^{i}}^{2} \\
= & \sum_{l=1}^{\infty} h_{l}^{-2} \sum_{s(k, i)=l}\left\|v-\widehat{Q}_{l} v\right\|_{0, \widetilde{\Omega}_{k}^{i}}^{2} \\
\lesssim & \sum_{l=1}^{\infty} h_{l}^{-2}\left\|v-\widehat{Q}_{l} v\right\|_{0, \Omega}^{2} \\
\lesssim & \|v\|_{a}^{2},
\end{aligned}
$$

where the last inequality is the stability of the multilevel decomposition for the general $L^{2}$ projection [21, 27]. 
Now from (10) and (14) we have

$$
\begin{aligned}
& \sum_{k=1}^{L} \sum_{i=1}^{n_{k}} h_{k, i}^{-2}\left\|\left(\Pi_{k}-\Pi_{k-1}\right) v\right\|_{0, \Omega_{k}^{i}}^{2} \\
= & \sum_{k=1}^{L} \sum_{i=1}^{n_{k}} h_{k, i}^{-2}\left\|\Pi_{k}\left(v-\widehat{Q}_{s(k, i)} v\right)-\Pi_{k-1}\left(v-\widehat{Q}_{s(k, i)} v\right)\right\|_{0, \Omega_{k}^{i}}^{2} \\
\leqslant & \sum_{k=1}^{L} \sum_{i=1}^{n_{k}} h_{k, i}^{-2}\left(\left\|\Pi_{k}\left(v-\widehat{Q}_{s(k, i)} v\right)\right\|_{0, \Omega_{k}^{i}}^{2}+\left\|\Pi_{k-1}\left(v-\widehat{Q}_{s(k, i)} v\right)\right\|_{0, \Omega_{k}^{i}}^{2}\right) \\
\lesssim & \sum_{k=1}^{L} \sum_{i=1}^{n_{k}} h_{k, i}^{-2}\left\|v-\widehat{Q}_{s(k, i)} v\right\|_{0, \widetilde{\Omega}_{k}^{i}}^{2} \lesssim\|v\|_{a}^{2}
\end{aligned}
$$

and

$$
\begin{aligned}
& \sum_{k=1}^{L} \sum_{i=1}^{n_{k}} h_{k, i}^{-2}\left\|v-\Pi_{k} v\right\|_{0, \Omega_{k}^{i}}^{2} \\
= & \sum_{k=1}^{L} \sum_{i=1}^{n_{k}} h_{k, i}^{-2}\left\|\left(v-\widehat{Q}_{s(k, i)} v\right)-\Pi_{k}\left(v-\widehat{Q}_{s(k, i)} v\right)\right\|_{0, \Omega_{k}^{i}}^{2} \\
\leqslant & \sum_{k=1}^{L} \sum_{i=1}^{n_{k}} h_{k, i}^{-2}\left(\left\|v-\widehat{Q}_{s(k, i)} v\right\|_{0, \Omega_{k}^{i}}^{2}+\left\|\Pi_{k}\left(v-\widehat{Q}_{s(k, i)} v\right)\right\|_{0, \Omega_{k}^{i}}^{2}\right) \\
\lesssim & \sum_{k=1}^{L} \sum_{i=1}^{n_{k}} h_{k, i}^{-2}\left\|v-\widehat{Q}_{s(k, i)} v\right\|_{0, \widetilde{\Omega}_{k}^{i}}^{2} \lesssim\|v\|_{a}^{2} .
\end{aligned}
$$

Finally, by the inverse inequality, we obtain

$$
\sum_{k=1}^{L}\left|\Pi_{k} v-\Pi_{k-1} v\right|_{1}^{2} \lesssim \sum_{k=1}^{L} \sum_{i=1}^{n_{k}} h_{k, i}^{-2}\left\|\left(\Pi_{k}-\Pi_{k-1}\right) v\right\|_{0, \Omega_{k}^{i}}^{2} \lesssim|v|_{1}^{2}
$$

\section{Estimate of convergence rate}

In this section we estimate $K$ in (7). $\forall v \in V$, we give a decomposition of $v$ and then estimate the numerator, denote by $K(v)$, of $K$ according to this decomposition. We prove that $K(v) \lesssim\|v\|_{a}^{2}$, hence $K \lesssim 1$ and we obtain the uniform convergence for our algorithm in Section 2.

The new projection proposed in Section 3 is applied to decompose $v$ into each level. By lemma 3.1, $\Pi_{k} v-\Pi_{k-1} v \in \sum_{i=1}^{n_{k}} V_{k}^{i}$, then we can decompose $v$ as follows:

$$
v=\sum_{k=1}^{L}\left(\Pi_{k}-\Pi_{k-1}\right) v=\sum_{k=1}^{L} \sum_{i=1}^{n_{k}} v_{k}^{i}, \quad v_{k}^{i} \in V_{k}^{i}
$$


Now we estimate

$$
K(v)=\sum_{k=1}^{L} \sum_{i=1}^{n_{k}}\left\|P_{k}^{i} \sum_{(l, j) \geqslant(k, i)} v_{l}^{j}\right\|_{a}^{2}
$$

with respect to the decomposition (15). Similar as the method developed in [22, 23], we split $\sum_{(l, j) \geqslant(k, i)} v_{l}^{j}$ into

$$
\begin{aligned}
\sum_{(l, j) \geqslant(k, i)} v_{l}^{j} & =\sum_{j=i}^{n_{k}} v_{k}^{j}+\sum_{l>k, j} v_{l}^{j} \\
& =\sum_{j=i}^{n_{k}} v_{k}^{j}+\sum_{l=k+1}^{L}\left(\Pi_{l}-\Pi_{l-1}\right) v \\
& =\sum_{j=i}^{n_{k}} v_{k}^{j}+\left(I-\Pi_{k}\right) v
\end{aligned}
$$

and then split $K(v)$ into two terms $K_{1}$ and $K_{2}$ as follows:

$$
\begin{aligned}
K(v) & \lesssim \sum_{k=1}^{L} \sum_{i=1}^{n_{k}}\left\|P_{k}^{i} \sum_{j=i}^{n_{k}} v_{k}^{j}\right\|_{a}^{2}+\sum_{k=1}^{L} \sum_{i=1}^{n_{k}}\left\|P_{k}^{i}\left(v-\Pi_{k} v\right)\right\|_{a}^{2} \\
& =K_{1}+K_{2},
\end{aligned}
$$

and then we estimate $K_{1}$ and $K_{2}$ respectively.

Firstly, by (11) in Lemma 3.7, we have

$$
\begin{aligned}
K_{1} & \lesssim \sum_{k=1}^{L} \sum_{i=1}^{n_{k}} h_{k, i}^{-2}\left\|v_{k}^{i}\right\|_{0}^{2} \\
& \lesssim \sum_{k=1}^{L} \sum_{i=1}^{n_{k}} h_{k, i}^{-2}\left\|\left(\Pi_{k}-\Pi_{k-1}\right) v\right\|_{0, \Omega_{k}^{i}}^{2} \\
& \lesssim\|v\|_{a}^{2}
\end{aligned}
$$

Next we split $K_{2}$ into two terms $K_{2,1}$ and $K_{2,2}$ as follows:

$$
\begin{aligned}
K_{2} & \lesssim \sum_{k=1}^{L} \sum_{i=1}^{n_{k}}\left\|P_{k}^{i}\left(v-\widehat{Q}_{s(k, i)} v\right)\right\|_{a}^{2}+\sum_{k=1}^{L} \sum_{i=1}^{n_{k}}\left\|P_{k}^{i}\left(\widehat{Q}_{s(k, i)} v-\Pi_{k} v\right)\right\|_{a}^{2} \\
& =K_{2,1}+K_{2,2}
\end{aligned}
$$


By (12) and (14), we obtain

$$
\begin{aligned}
K_{2,2} & \lesssim \sum_{k=1}^{L} \sum_{i=1}^{n_{k}}\left|\widehat{Q}_{s(k, i)} v-\Pi_{k} v\right|_{1, \Omega_{k}^{i}}^{2} \\
& \lesssim \sum_{k=1}^{L} \sum_{i=1}^{n_{k}} h_{k, i}^{-2}\left\|\widehat{Q}_{s(k, i)} v-\Pi_{k} v\right\|_{0, \Omega_{k}^{i}}^{2} \\
& \lesssim \sum_{k=1}^{L} \sum_{i=1}^{n_{k}} h_{k, i}^{-2}\left\|v-\Pi_{k} v\right\|_{0, \Omega_{k}^{i}}^{2}+\sum_{k=1}^{L} \sum_{i=1}^{n_{k}} h_{k, i}^{-2}\left\|v-\widehat{Q}_{s(k, i)} v\right\|_{0, \Omega_{k}^{i}}^{2} \\
& \lesssim\|v\|_{a}^{2} .
\end{aligned}
$$

Finally we estimate $K_{2,1}$. Firstly we have

$$
\begin{aligned}
K_{2,1} & =\sum_{k=1}^{L} \sum_{i=1}^{n_{k}} a\left(P_{k}^{i}\left(v-\widehat{Q}_{s(k, i)} v\right), v-\widehat{Q}_{s(k, i)} v\right) \\
& =\sum_{k=1}^{L} \sum_{i=1}^{n_{k}} \sum_{l>s(k, i)} a\left(P_{k}^{i}\left(v-\widehat{Q}_{s(k, i)} v\right), \widehat{Q}_{l} v-\widehat{Q}_{l-1} v\right) .
\end{aligned}
$$

By the strengthened Cauchy-Schwartz inequality $([21,27])$ on $\Omega_{k}^{i}$, we obtain

$$
\begin{aligned}
& a\left(P_{k}^{i}\left(v-\widehat{Q}_{s(k, i)} v\right), \widehat{Q}_{l} v-\widehat{Q}_{l-1} v\right) \\
\lesssim & \gamma^{l-s(k, i)}\left\|P_{k}^{i}\left(v-\widehat{Q}_{s(k, i)} v\right)\right\|_{a} h_{l}^{-1}\left\|\widehat{Q}_{l} v-\widehat{Q}_{l-1} v\right\|_{0, \Omega_{k}^{i}},
\end{aligned}
$$

where $\gamma$ is the quasi-uniform parameter of the meshes $\widehat{V}_{l}$ with $0<\gamma<1$. By (16) and (17),

$$
\begin{aligned}
& K_{2,1} \\
\lesssim & \sum_{m \geqslant 1} \gamma^{m} \sum_{k=1}^{L} \sum_{i=1}^{n_{k}}\left\|P_{k}^{i}\left(v-\widehat{Q}_{s(k, i)} v\right)\right\|_{a} h_{s(k, i)+m}^{-1}\left\|\widehat{Q}_{s(k, i)+m} v-\widehat{Q}_{s(k, i)+m-1} v\right\|_{0, \Omega_{k}^{i}} \\
\lesssim & \sum_{m \geqslant 1} \gamma^{m}\left(K_{2,1}\right)^{\frac{1}{2}} \cdot\left(\sum_{k=1}^{L} \sum_{i=1}^{n_{k}} h_{s(k, i)+m}^{-2}\left\|\widehat{Q}_{s(k, i)+m} v-\widehat{Q}_{s(k, i)+m-1} v\right\|_{0, \Omega_{k}^{i}}^{2}\right)^{\frac{1}{2}} \\
\lesssim & \sum_{m \geqslant 1} \gamma^{m}\left(K_{2,1}\right)^{\frac{1}{2}} \cdot\left(\sum_{l=1}^{\infty} h_{l}^{-2}\left\|\widehat{Q}_{l} v-\widehat{Q}_{l-1} v\right\|_{0}^{2}\right)^{\frac{1}{2}} \\
\lesssim & \left(K_{2,1}\right)^{\frac{1}{2}} \cdot\left(\|v\|_{a}^{2}\right)^{\frac{1}{2}}
\end{aligned}
$$

where the last inequality is the stability of the multilevel decomposition for the general $L^{2}$ projection $[21,27]$. Therefore

$$
K_{2,1} \lesssim\|v\|_{a}^{2}
$$

Now we have proved $K(v) \lesssim\|v\|_{a}^{2}$ and the uniform convergence of our algorithm. 


\section{5. high order Lagrange element}

In this section we consider the discretization of the high order Lagrange element. The setting of the meshes is similar as the piecewise linear element. Suppose we have a sequence of nested triangulations $\mathcal{T}_{k}=\left\{\tau_{k}^{i}\right\}\left(1 \leq k \leq L, 1 \leq i \leq p_{k}\right)$ of $\Omega$, where $\tau_{k}^{i}$ is simplex. Also the requirement on $\mathcal{T}_{k}$ is (A1), (A2) and (A3).

Similarly we denote by $V_{k}\left(\subset H_{0}^{1}(\Omega)\right)$ the finite element space of the continuous piecewise linear functions associated with triangulation $\mathcal{T}_{k}$, and the difference is the definition of $V$. Here we denote by $V$ the high order Lagrange finite element space associated with the finest mesh $\mathcal{T}_{L}$. Then

$$
V_{1} \subset \cdots \subset V_{k} \subset \cdots \subset V_{L} \subset V .
$$

and the discrete problem is: find $u \in V$ such that

$$
a(u, v)=(f, v), \quad \forall v \in V .
$$

The definition of nodal basis function $\phi_{k}^{i}$ and its space $V_{k}^{i}$ is same as the definition in Section 2. Note that $V_{L}^{i}$ is only the space of nodal basis of $V_{L}$ but not the space of nodal basis of $V$ in this section. In order to introduce the space of nodal basis of $V$, we denote by $\phi_{L L}^{i}$ the nodal basis of $V$ and define $V_{L L}^{i}=\operatorname{span}\left\{\phi_{L L}^{i}\right\}$ with $1 \leq i \leq m$. Similarly, we can define $P_{L L}^{i}, \Omega_{L L}^{i}, h_{L L, i}, \widetilde{\Omega}_{L L}^{i}$ and so on.

Now the finite element space $V$ can be decompose as

$$
V=\sum_{i=1}^{m} V_{L L}^{i}+\sum_{k=1}^{L} \sum_{i=1}^{n_{k}} V_{k}^{i}
$$

According to the decomposition (19), Algorithm 2.1 gives the multigrid algorithm for the high order Lagrange element: we perform relaxation on all the nodes of the only finest grid, then the method for other levels is like that in the linear finite element. To prove the uniform convergence of this algorithm, similarly $\forall v \in V$, we decompose $v$ as follows:

$v=\left(v-\Pi_{L} v\right)+\sum_{k=1}^{L}\left(\Pi_{k}-\Pi_{k-1}\right) v=\sum_{i=1}^{m} v_{L L}^{i}+\sum_{k=1}^{L} \sum_{i=1}^{n_{k}} v_{k}^{i}, \quad v_{L L}^{i} \in V_{L L}^{i}, \quad v_{k}^{i} \in V_{k}^{i}$.

And then we estimate

$$
K=\sup _{v \in V \sum_{i=1}^{m} v_{L L}^{i}+\sum_{k=1}^{L} \sum_{i=1}^{n_{k}} v_{k}^{i}=v} \frac{K(v)}{\|v\|_{a}^{2}}
$$

where

$$
\begin{aligned}
K(v) & =\sum_{i=1}^{m}\left\|P_{L L}^{i}\left(\sum_{j=i}^{m} v_{L L}^{j}+\sum_{k=1}^{L} \sum_{i=1}^{n_{k}} v_{k}^{i}\right)\right\|_{a}^{2}+\sum_{k=1}^{L} \sum_{i=1}^{n_{k}}\left\|P_{k}^{i} \sum_{(l, j) \geqslant(k, i)} v_{l}^{j}\right\|_{a}^{2} \\
& =\sum_{i=1}^{m}\left\|P_{L L}^{i}\left(\sum_{j=i}^{m} v_{L L}^{j}+\Pi_{L} v\right)\right\|_{a}^{2}+\sum_{k=1}^{L} \sum_{i=1}^{n_{k}}\left\|P_{k}^{i} \sum_{(l, j) \geqslant(k, i)} v_{l}^{j}\right\|_{a}^{2} .
\end{aligned}
$$


First we have

$$
\begin{aligned}
& \sum_{i=1}^{m}\left\|P_{L L}^{i}\left(\sum_{j=i}^{m} v_{L L}^{j}+\Pi_{L} v\right)\right\|_{a}^{2} \\
\leq & \sum_{i=1}^{m}\left\|P_{L L}^{i}\left(\sum_{j=i}^{m} v_{L L}^{j}\right)\right\|_{a}^{2}+\sum_{i=1}^{m}\left\|P_{L L}^{i}\left(\Pi_{L} v\right)\right\|_{a}^{2} \\
\lesssim & \sum_{i=1}^{m}\left|\sum_{j=i}^{m} v_{L L}^{j}\right|_{1, \Omega_{L L}^{i}}^{2}+\sum_{i=1}^{m}\left|\Pi_{L} v\right|_{1, \Omega_{L L}^{i}}^{2} \\
\lesssim & \sum_{i=1}^{m}\left|v_{L L}^{i}\right|_{1}^{2}+\left|\Pi_{L} v\right|_{1}^{2} \\
\lesssim & \sum_{i=1}^{m} h_{L L, i}^{-2}\left\|v_{L L}^{i}\right\|_{0}^{2}+\left|\Pi_{L} v\right|_{1}^{2} \\
\lesssim & \sum_{i=1}^{m} h_{L L, i}^{-2}\left\|v-\Pi_{L} v\right\|_{0, \Omega_{L L}^{i}}^{2}+\left|\Pi_{L} v\right|_{1}^{2} \\
\lesssim & \sum_{i=1}^{m}|v|_{1, \widetilde{\Omega}_{L L}^{i}}^{2}+\left|\Pi_{L} v\right|_{1}^{2}\left(B y L_{L} m m a 3.6\right) \\
\lesssim & |v|_{1}^{2}+\left|\Pi_{L} v\right|_{1}^{2},
\end{aligned}
$$

and from Section 4, we obtain

$$
\sum_{k=1}^{L} \sum_{i=1}^{n_{k}}\left\|P_{k}^{i} \sum_{(l, j) \geqslant(k, i)} v_{l}^{j}\right\|_{a}^{2} \lesssim\left|\Pi_{L} v\right|_{1}^{2},
$$

additional by lemma 3.4, we have $\left|\Pi_{L} v\right|_{1}^{2} \lesssim|v|_{1}^{2}$, then

$$
K(v) \lesssim|v|_{1}^{2}+\left|\Pi_{L} v\right|_{1}^{2}+\left|\Pi_{L} v\right|_{1}^{2} \lesssim|v|_{1}^{2}=\|v\|_{a}^{2}
$$

therefore $K \lesssim 1$ and we have proved the uniform convergence of the algorithm for high order Lagrange element.

\section{6. numerical results}

In this section, we present some numerical results to illustrate our conclusion. Our numerical results are based on the MATLAB package $i$ FEM [24]. In the adaptive procedure, new vertex bisection is used in the $2 \mathrm{D}$ case and longest edge bisection is used in the 3D case. According to the adaptive process, on each refined mesh we need solve a discrete problem. In our numerical practice, we use the multigrid method which is described in this paper to solve each discrete problem. The stop criterion of the multigrid iteration is that the residual has been reduced below $10^{-8}$. Then this multigrid solution is applied to estimate the a posteriori error and then the elements with largest error indicators are marked and refined. After each refinement, a new discrete 
problem is appeared according to the new grid, and we apply the multigrid iteration in this new loop. We apply our method in two problems in 2D and two problems in 3D. In each example, we choose some certain adaptive grid levels and present information on these levels, including the degree of freedoms, the iterations when the residual has been reduced below $10^{-8}$ and then the multigrid iteration stops, the CPU time cost to solve the discrete problem on this level, the $L^{2}$ norm and energy norm error between the discrete solution and the actual solution on this level.

The first example in 2D is the crack problem, the domain is $\{|x|+|y|<1\} \backslash[0,1) \times$ $\{0\}$ and the actual solution is set to be $u(r, \theta)=r^{1 / 2} \sin \frac{\theta}{2}-\frac{1}{4} r^{2}$. Figure 1 gives the adaptive mesh of the 10-th level. The numerical results are shown in Table 1.

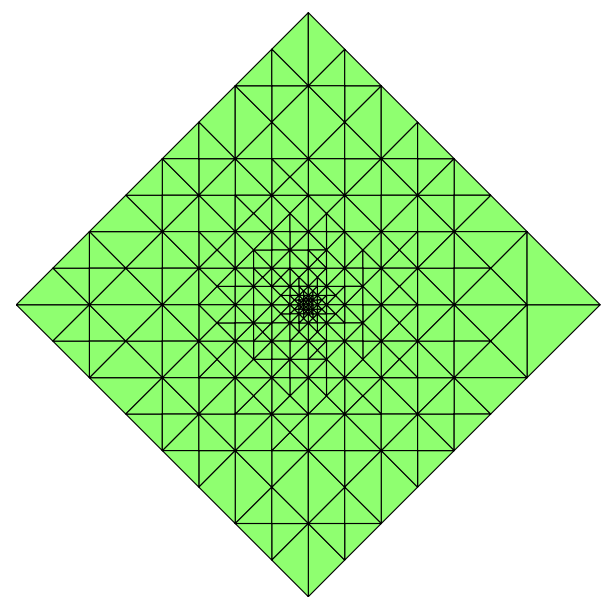

Figure 1: Example 1 in 2D case

Table 1: results of example 1 in $2 \mathrm{D}$ case

\begin{tabular}{|c|c|c|c|c|c|c|}
\hline level & 24 & 26 & 28 & 30 & 32 & 34 \\
\hline DOFs & 12191 & 21711 & 38268 & 67690 & 119631 & 212664 \\
\hline iterations & 9 & 9 & 10 & 10 & 10 & 10 \\
\hline$L^{2}$ error & $8.59 \times 10^{-5}$ & $5.13 \times 10^{-5}$ & $3.33 \times 10^{-5}$ & $1.53 \times 10^{-5}$ & $1.03 \times 10^{-5}$ & $4.66 \times 10^{-6}$ \\
\hline$H^{1}$ error & $1.51 \times 10^{-2}$ & $1.13 \times 10^{-2}$ & $8.44 \times 10^{-3}$ & $6.33 \times 10^{-3}$ & $4.72 \times 10^{-3}$ & $3.52 \times 10^{-3}$ \\
\hline CPU time & 0.24 & 0.42 & 0.76 & 1.4 & 2.5 & 4.8 \\
\hline
\end{tabular}

The second example in $2 \mathrm{D}$ is the $L$-shape problem, the domain is $(-1,1) \times(-1,1) \backslash[0,1) \times$ $(-1,0]$ and the actual solution is set to be $u(r, \theta)=r^{2 / 3} \sin \frac{2 \theta}{3}$. Figure 2 gives the adaptive mesh of the 10-th level. The numerical results are shown in Table 2. 


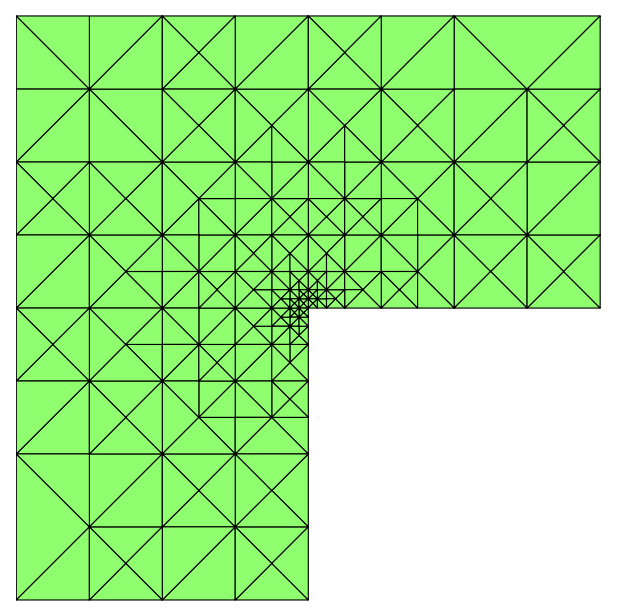

Figure 2: Example 2 in 2D case

Table 2: results of example 2 in $2 \mathrm{D}$ case

\begin{tabular}{|c|c|c|c|c|c|c|}
\hline level & 25 & 27 & 29 & 31 & 33 & 35 \\
\hline DOFs & 13860 & 24214 & 42283 & 73403 & 126765 & 219241 \\
\hline iterations & 8 & 8 & 8 & 8 & 8 & 8 \\
\hline$L^{2}$ error & $2.03 \times 10^{-5}$ & $1.15 \times 10^{-5}$ & $7.25 \times 10^{-6}$ & $3.56 \times 10^{-6}$ & $2.56 \times 10^{-6}$ & $1.25 \times 10^{-6}$ \\
\hline$H^{1}$ error & $7.18 \times 10^{-3}$ & $5.40 \times 10^{-3}$ & $4.08 \times 10^{-3}$ & $3.09 \times 10^{-3}$ & $2.35 \times 10^{-3}$ & $1.78 \times 10^{-3}$ \\
\hline CPU time & 0.23 & 0.42 & 0.72 & 1.3 & 2.2 & 4.0 \\
\hline
\end{tabular}


The first example in $3 \mathrm{D}$ is the cube problem, the domain is $(-1,1) \times(-1,1) \times$ $(-1,1)$ and the actual solution is set to be $u(x, y, z)=e^{-10\left(x^{2}+y^{2}+z^{2}\right)}$. Figure 3 gives the adaptive mesh of the 10-th level on the section of $z=0$. The numerical results are shown in Table 3.

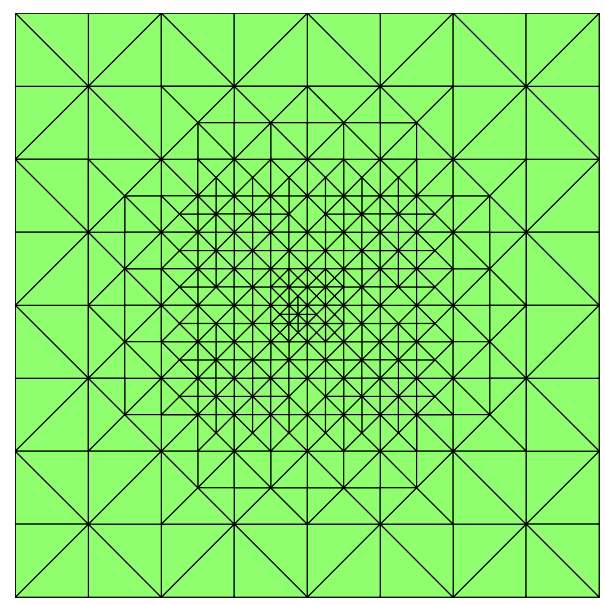

Figure 3: Example 1 in 3D case

Table 3: results of example 1 in 3D case

\begin{tabular}{|c|c|c|c|c|c|c|}
\hline level & 14 & 15 & 16 & 17 & 18 & 19 \\
\hline DOFs & 23648 & 36349 & 55600 & 85059 & 130077 & 198816 \\
\hline iterations & 15 & 13 & 15 & 15 & 14 & 16 \\
\hline$L^{2}$ error & $1.52 \times 10^{-3}$ & $1.18 \times 10^{-3}$ & $8.98 \times 10^{-4}$ & $6.66 \times 10^{-4}$ & $4.97 \times 10^{-4}$ & $3.83 \times 10^{-4}$ \\
\hline$H^{1}$ error & $1.13 \times 10^{-1}$ & $9.95 \times 10^{-2}$ & $8.67 \times 10^{-2}$ & $7.38 \times 10^{-2}$ & $6.30 \times 10^{-2}$ & $5.51 \times 10^{-2}$ \\
\hline CPU time & 0.78 & 1.1 & 1.8 & 2.8 & 4.3 & 7.2 \\
\hline
\end{tabular}

The second example in $3 \mathrm{D}$ is the hollow cube problem, the domain is $(-1,2) \times$ $(-1,2) \times(-1,2) \backslash[0,1] \times[0,1] \times[0,1]$ and the actual solution is set to be $u(x, y, z)=$ $e^{-10\left(x^{2}+y^{2}+z^{2}\right)}$. Figure 4 gives the adaptive mesh of the 10-th level on the section of $z=0$. The numerical results are shown in Table 4 .

From the numerical practice, first we can see the optimality for the adaptive method: the $L^{2}$ error is inverse proportional to the degree of freedoms and the energy norm is inverse proportional to the square root of the degree of freedoms. For the multigrid method, we see that the iterations needed on each level is not increased, which illustrates the uniform convergence of our algorithm. Our numerical experiments also show 


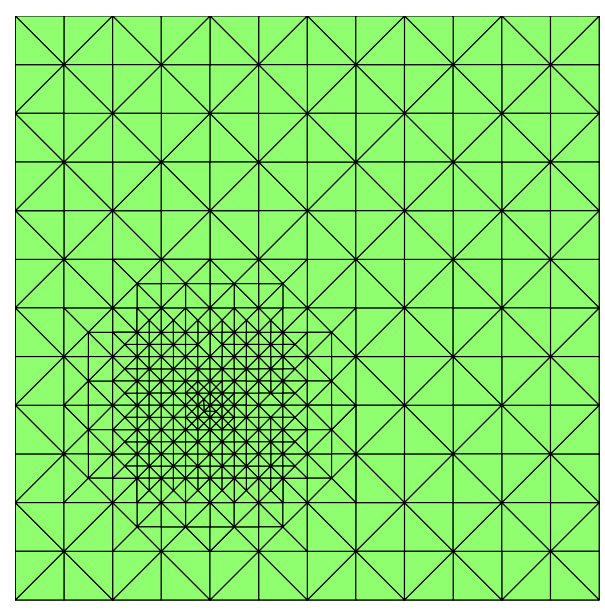

Figure 4: Example 2 in 3D case

Table 4: results of example 2 in $3 \mathrm{D}$ case

\begin{tabular}{|c|c|c|c|c|c|c|}
\hline level & 15 & 16 & 17 & 18 & 19 & 20 \\
\hline DOFs & 33920 & 51190 & 77427 & 117159 & 178298 & 268288 \\
\hline iterations & 12 & 12 & 12 & 12 & 13 & 13 \\
\hline$L^{2}$ error & $1.03 \times 10^{-3}$ & $7.69 \times 10^{-4}$ & $5.72 \times 10^{-4}$ & $4.34 \times 10^{-4}$ & $3.36 \times 10^{-4}$ & $2.49 \times 10^{-4}$ \\
\hline$H^{1}$ error & $9.31 \times 10^{-2}$ & $8.10 \times 10^{-2}$ & $6.89 \times 10^{-2}$ & $5.89 \times 10^{-2}$ & $5.15 \times 10^{-2}$ & $4.55 \times 10^{-2}$ \\
\hline CPU time & 0.95 & 1.5 & 2.3 & 3.5 & 5.8 & 9 \\
\hline
\end{tabular}


the multigrid method's optimality on computational complexity that the computational time cost is proportional to the degree of freedoms.

\section{References}

[1] W. Dorfler, A convergent adaptive algorithm for poisson's equation, SIAM J. Numer. Anal. 33 (1996) 1106-1124.

[2] P. Morin, R. H. Nochetto, K. G. Siebert, Convergence of adaptive finite element methods, SIAM Rev. 44 (2002) 631-658.

[3] K. Mekchay, R. H. Nochetto, Convergence of adaptive finite element methods for general second order linear elliptic PDE, SIAM J. Numer. Anal. 43 (2005) $1803-1827$.

[4] P. Binev, W. Dahmen, R. DeVore, Adaptive finite element methods with convergence rates, Numer. Math. 97 (2004) 219-268.

[5] R. Stevenson, Optimality of a standard adaptive finite element method, Found. Comput. Math. 7 (2007) 245-269.

[6] J. M. Cascon, C. Kreuzer, R. H. Nochetto, K. G. Siebert, Quasi-optimal convergence rate for an adaptive finite element method, SIAM J. Numer. Anal. 46 (2008) 2524-2550.

[7] D. Bai, A. Brandt, Local mesh refinement multilevel techniques, SIAM J. Sci. Stat. Comp. 8 (1987) 109-134.

[8] A. Brandt, Multi-level adaptive solutions to boundary-value problem, Math. Comput. 31 (1977) 333-390.

[9] S. McCormick, J. Thomas, The fast adaptive composite grid (fac) method for elliptic equations, Math. Comput. 46 (1986) 439-456.

[10] S. McCormick, Fast adaptive composite grid (FAC) methods: theory for the variational case. In: Defect Correction Methods (Oberwolfach, 1983), volume 5 of Comput. Suppl., Springer, Vienna, 1984.

[11] P. Bastian, C. Wieners, Multigrid methods on adaptively refined grids, Computing in science engineering 8 (2006) $44-54$.

[12] J. H. Bramble, R. E. Ewing, J. E. Pasciak, A. H. Schatz, A preconditioneding technique for the efficient solution of problems with local grid refinement, Comput. Methods Appl. Mech. Engrg. 67 (1988) 149-159.

[13] M. Ainsworth, W. Mclean, Multilevel diagonal scaling preconditioners for boundary element equations on locally refined meshes, Numer. Math. 93 (2003) 387-413.

[14] R. E. Bank, T. Dupont, H. Yserentant, The hierarchical basis multigrid method, Numer. Math. 52 (1988) 427-458. 
[15] H. Yserentant, Two preconditioners based on the multi-level splitting of fnite element spaces, Numer. Math. 58 (1990) 163-184.

[16] W. F. Mitchell, Optimal multilevel iterative methods for adaptive grids, SIAM J. Sci. Stat. Comput. 13 (1992) 146-167.

[17] $\mathrm{H}$. Wu, Z. Chen, Uniform convergence of multigrid v-cycle on adaptively refined finite element meshes for second order elliptic problems, Science in China: Series A Mathematics 49 (2006) 1-28.

[18] L. Chen, R. H. Nochetto, J. Xu, Optimal multilevel methods for graded bisection grids, Numer. Math. 120 (2012) 1-34.

[19] J. Xu, L. Zikatanov, The method of alternating projections and the method of subspace corrections in hilbert space, J. Am. Math. Soc. 15 (2002) 573-597.

[20] R. Scott, S. Zhang, Finite element interpolation of nonsmooth functions satisfying boundary conditions, Math. Comput. 54 (1990) 483-493.

[21] J. Xu, Iterative methods by space decomposition and subspace correction, SIAM Rev. 34 (1992) 581-613.

[22] Y. J. Lee, J. Wu, J. Xu, L. Zikatanov, A sharp convergence estimate of the method of subspace corrections for singular systems, Math. Comp. 77 (2008) 831-850.

[23] Y. J. Lee, J. Wu, J. Chen, Robust multigrid methods for the planar linear elasticity problems, Numer. Math. 113 (2009) 473-496.

[24] L. Chen, an integrate finite element methods package inMATLAB, Technical Report, University of California at Irvine, 2009.

[25] I. Kossaczký, A recursive approach to local mesh refinement in two and three dimensions, J. Comput. Appl. Math. 55 (1994) 275-288.

[26] R. Stevenson, The completion of locally refined simplicial partitions created by bisection, Math. Comput. 77 (2008) 227-241.

[27] J. Xu, An introduction to multigrid convergence theory. In: Iterative Methods in Scientific Computing, Springer, Berlin, 1997. 